\title{
Scheduling Customized Orders by Considering the Ergonomic Constraints: A Case Study at YEMTAR Company
}

\author{
A. Deniz Karaoglan'1, M. A. Beyazit Ocaktan ${ }^{1}$, Demet Gonen ${ }^{1}$, Ali Oral ${ }^{2}$, S. Selin Kundakci ${ }^{3}$, Can Tuncer ${ }^{3}$ \\ ${ }^{1}$ Department of Industrial Engineering, Balikesir University \\ 10145 Cagis Campus, Balikesir, Turkey \\ deniz@balikesir.edu.tr, ocaktan@balikesir.edu.tr, dgonen@balikesir.edu.tr \\ ${ }^{2}$ Department of Mechanical Engineering, Balikesir University \\ 10145 Cagis Campus, Balikesir, Turkey \\ alioral@balikesir.edu.tr \\ ${ }^{3}$ Research \& Development Department, YEMTAR \\ 50 10200, Bandirma, Balikesir, Turkey \\ skundakci@yemtar.com, ctuncer@yemtar.com
}

\begin{abstract}
It is important for companies to meet customer demands by due date and reduce the labor cost on the finalized product. For this purpose, order scheduling is required for different purposes such as minimizing makespan, maximizing resource utilization, etc. Dynamic production environment causes stochastic operation times at companies which work based on project type labor-intensive production. Stochastic operation times make order scheduling harder. There are many reasons that causes operation times being stochastic such as technical specifications of the orders, skills of the operators, bottlenecks in the job-shop, and etc. However, one of the most important but less discussed constraints that affect the probability distribution of the operation times is the ergonomic constraint. Ergonomic constraints, such as musculoskeletal discomfort, fatigue and limitations determined by the laws make it even more difficult to predict the total makespan of waiting orders. In this study, an order scheduling algorithm that considers the dynamical production environment and the ergonomic limitations is proposed for nearly optimizing average makespan for several waiting orders in the grinding and painting workstation of YEMTAR Company. The proposed algorithm adopts the technical order specifications and ergonomic constraints together, computes the stochastic operation times by using simulation, and schedule orders by using genetic algorithm. The objective is to determine the entry sequence of the waiting orders to the workshop for minimizing their average makespan which directly influences the resource utilization, efficiency, and labor costs.
\end{abstract}

Keywords: Order Scheduling, Ergonomic Constraints, Makespan, Genetic Algorithm, Simulation.

\section{Introduction}

Order scheduling is used for determining the entry sequence of the waiting orders to the workshop and required for minimizing the labor cost, due date, makespan, and etc. Makespan is one of the most important performance measurements of the order scheduling and defined as the total time elapses from the beginning to the end for the given orders set. In other words, it is the time difference between the start and finish of a sequence of job or tasks. In the labor intensive project type manufacturing; except a few similar orders produced frequently; there are unlimited types of different orders varying based on the technical specifications demanded by the customers and the state of the shop floor is changed continuously. This variation causes unknown processing times for each type of order which makes order scheduling harder [1-5].

In this study, an order scheduling algorithm that considers the dynamical production environment, technical specifications of orders demanded by the customer and the ergonomic limitations related by these technical specifications is proposed. The case study is performed for nearly optimizing average makespan for several waiting orders of a feed machine producer namely YEMTAR company. In the YEMTAR, the manufacturing is performed by project type production. For this reason, the conventional scheduling approach that only considers the general job and shop characteristics (the number of machines, the number of jobs in the queue, etc.) are insufficient. In such a production system, each of the received order can be considered as a new product. The orders may be completely different because of their technical specifications demanded by the customer $[1,3]$. 
The production operations of YEMTAR consist of five main steps; (i) machining, (ii) cutting and bending, (iii) welding, (iv) grinding and painting, and (v) final assembly. This study is focused on order scheduling for the grinding and painting unit. In the proposed algorithm, genetic algorithm (GA) is used for scheduling orders under comprehensive design constraints. Arena simulation is used to calculate processing times and makespan of operations based on order sequences generated by GA. The simulation model uses probability distributions as input to model according to technical design parameters of orders and ergonomic constraints to calculate processing times.

Although various GA approaches have been implemented for solving job-shop scheduling problem in the literature [611], the technical specifications of orders and the ergonomic constraints related with them are considered in none of them. Ergonomic constraints are considered in solving job shop scheduling in the literature. Baykasoglu et al. [12], argued that ergonomics has been playing an important role in assembly system design (ASD). ASD contains not only the main assembly line balancing problem, but also the subassembly line balancing and assembly layout problem. The ergonomics in ASD has an impact both on productivity and on workers' health, especially when frequent changes in the product mix occur. In this study, they proposed a systematic approach in order to handle ASD, which consists of ergonomic risks and layout problem. Sebnem et al. [13] proposed a new type of assembly line worker assignment and balancing problem (ALWABP) which considers ergonomic risks. ALWABP occurs when task times vary according to the assigned worker. Although the operation time of a task is assumed to be fixed in classical assembly lines, it depends on the operator who executes the task in ALWABP. Carnahan et al. [14], proposed the ergonomic job rotation scheduling problem (EJRSP), which aims at smoothing ergonomic risks between workers by minimizing the ergonomic load for the worker exposed to ergonomic risks. They used genetic algorithm to solve EJRSP and used a neural network algorithm to find promising rules for job rotation. Kullpattaranirun and Nanthavanij[15], presented a heuristic genetic algorithm (GA) to find the work assignments for a group of workers in which the maximum daily noise exposure level that any of the workers exposed is minimized. Aryanezhad et al. [16] developed a new model considering two objective functions simultaneously. The first objective function aims to minimize maximum occupational noise exposure injuries, and the second one is designed to minimize the potential of worker's low back injuries. This model deals with safe skill-based job rotation scheduling (SSJRS). Kara et al. [17] proposed a new cost-based model that constrains psychological strain, physical strain, working skills, multiple operators, equipment, working postures, and lighting levels in their work. However, none of these studies considered technical specifications of orders and ergonomic constraints together. The following section gives a brief description of the material and methods used in this study.

\section{Proposed Method}

In this study GA is used to find the best sequence of customer orders, which minimizes average makespan, and Arena simulation is used to calculate processing times and makespan of operations based on order sequences generated by GA. Please refer to $[1,3,18,19]$ for a detailed discussion on the problem and also refer to [20,21] for a detailed discussion on GA.

Simulation is "the imitation of the operation of a real world process or system over time" and enables the study of and experimentation with the internal interactions of a complex system $[1,22]$. In a simulation work, numerical experiments are conducted on digital computer and it can be used a general-purposed programming language or a special-purposed simulation programming language. There are many simulation-purposed languages such as Arena, Promodel etc. for conducting a simulation experiment. Arena exploits ActiveX Automation and Visual Basic for Applications (VBA) for integrating directly with other programs such as Microsoft Office. In this paper, Arena 14 simulation software is used for the simulation [1, 3]. The proposed GA is coded in VBA environment which makes connection to Arena simulation software hassle-free. All GA specific components are implemented in VBA. Arena is used for computing the fitness value $(\mathrm{FV})$ of each chromosome via simulating the complex system. The system here corresponds to the dynamic labor-intensive project-type grinding and painting workstations of YEMTAR production system including various operations, queues and capacity constraints of which the details will be provided in the next section.

Figure 1 depicts the graphical summary of the proposed GA-based optimization approach to minimize average makespan, namely, the total completion time of all customer orders. GA is used here for tuning the sequence of waiting customized orders for importing them to the system. The algorithm starts with randomly generating the initial population, which involves PopSize number of chromosomes. Each chromosome is made up of a permuted sequence of orders from 1 to the total number of orders, where each gene corresponds to an order number. 


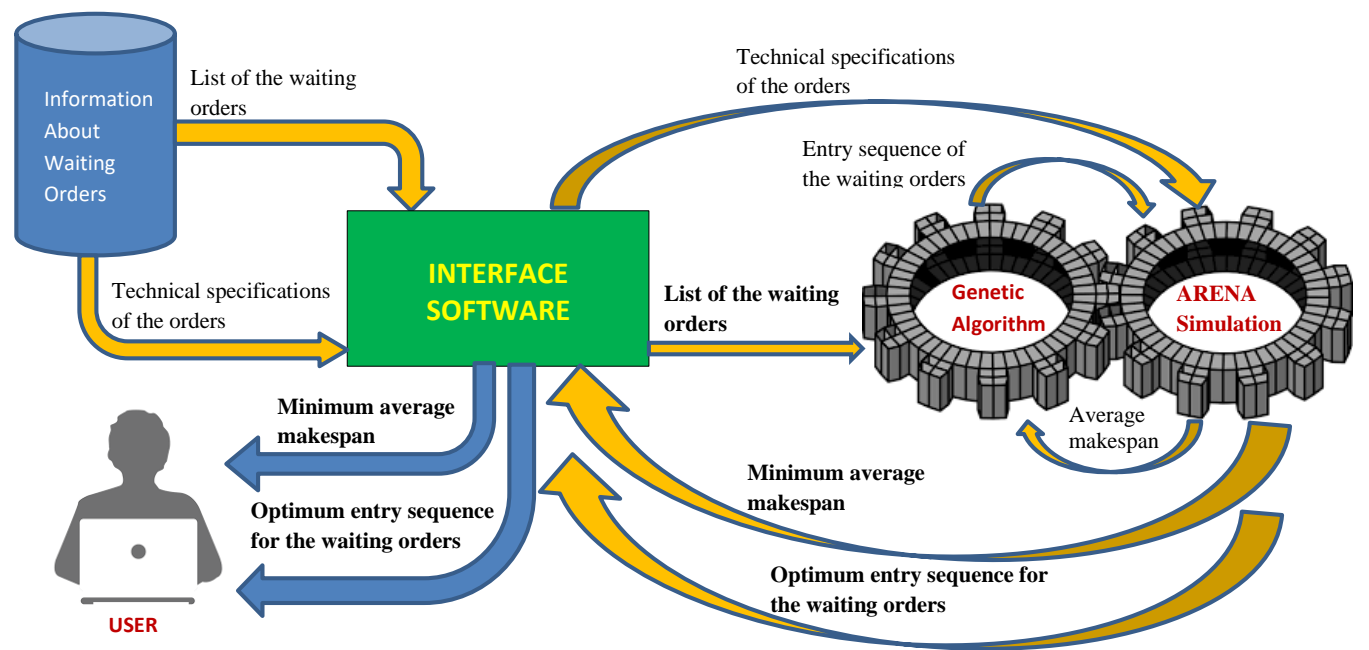

Fig. 1: The graphical summary of the proposed simulation based genetic algorithm approach.

As seen from the figure, each order is shown only once on the chromosome. This is a sample complete solution for our problem. Orders are transferred to the production system in which they are shown on the chromosome consisting of 20 orders given in Figure 2, i.e. 14, 11, 6, and so on.

\begin{tabular}{|l|l|l|l|l|l|l|l|l|l|l|l|l|l|l|l|l|l|l|l|}
\hline 14 & 11 & 6 & 18 & 19 & 1 & 2 & 5 & 7 & 8 & 10 & 12 & 3 & 4 & 17 & 15 & 20 & 9 & 13 & 16 \\
$\underbrace{}$
\end{tabular}

Fig. 2: The representation of a sample chromosome.

Pseudo-code of proposed simulation based genetic algorithm for order scheduling that is graphically presented in Figure 1 is given below [1]:

\section{START}

(Step 1) READ the list of waiting orders and their technical specifications from the EXCEL file where these data are recorded

(Step 2) DETERMINE the genetic algorithm parameters (population size: PopSize, crossover rate ( $c r$ ), mutation rate $(\mathrm{mr})$, maximum number of iterations (MaxNbIt))

(Step 3) GENERATE initial population for the genetic algorithm (PopSize)

(the order sequence represents the chromosomes and the each waiting order at the list represents the genes)

(Step 4) CALCULATE fitness values (FV) of all chromosomes in the initial population by running ARENA Simulation

Step 4.1 RUNArena Simulation for the selected chromosomes (order sequence of waiting jobs) and calculate the FV values (where FV is the average makespan calculated by ARENA for the selected order sequence)

Step 4.2 IF FVs of all chromosomes in the initial population are computed. GO TO Step 5

(Step 5) GENERATE new individuals (new order sequences: chromosomes) for providing diversity

Step 5.1 USE roulette wheel selection method to select chromosomes for crossover and mutation.

- CALCULATE the probability $P(i)$ of selecting the $i$-th individual by $P(i)=\frac{1 / F V(i)}{\sum_{k=1}^{\text {Popsize }} 1 / F V(k)}$

- DETEMINE the number of chromosomes to apply crossover by the multiplication of PopSize and crossover rate $(c r)$

- APPLY one-point crossover by determining a random number between 1 and PopSize-1 (which is called the cutting point $(c p))$ 
- DETEMINE the number of chromosomes to apply mutation by the multiplication of PopSize and mutation rate $(m r)$

- APPLY mutation by using swap method or insert method

(For each selected chromosome, a random number $(r n d)$ is determined between 0 and 1 and if $r n d<0.5$, swap is applied; otherwise, insert is applied.)

Step 5.2 CALCULATE fitness values (FV) of all child chromosomes in the initial population by running ARENA Simulation

Step 5.3 DO the once which have better $F V s$ are included in the population replacing worse ones

Step 5.4 IF MaxNbIt is reached, THEN GO TO Step 6 ELSE RETURN TO Step 5.1

(Step 6) TERMINATE the simulation based genetic algorithm

(Step 7) REPORT the optimum entry sequence to the workshop for the waiting orders and the minimum average makespan for the waiting orders.

\section{Case Study}

\subsection{Production System of YEMTAR Company and the Problem Definition}

YEMTAR is a feed machine producer located in Balikesir - Bandirma, Turkey. Also, the company is establishing turnkey feed mill systems. The company produces mills, pellet presses, mixers, grinding lines, elevators, conveyors, rendering units, premix units, flaking plants, dosing and micro dosing lines, fertilizer Plants in $12000 \mathrm{~m}^{2}$ closed area, 34000 $\mathrm{m}^{2}$ total production area. The production operations of a YEMTAR consist of five main steps, (i) machining, (ii) cutting and bending, (iii) welding, (iv) grinding and painting, and (v) final assembly. This study is focused on order scheduling for the grinding and painting unit. The manufacturing line is designed as a labor-intensive project-type production, composed of sequential processes including the grinding and painting operations. In grinding operation, different components of orders those are welded in the welding operation are grinded. After completion of grinding operation, these components are painted in the painting station. The processing time of grinding is affected from the weight of the part $\left(X_{1}\right)$ (1: heavy, 2: light), being bolted or welded $\left(X_{2}\right)$ (1: bolted, 2: welded), size $\left(X_{3}\right)$ (1: small, 2: big) of the part to be grinded, suitability at working on grinding table or working on ground $\left(X_{4}\right)$ (1: table, 2: ground), transportation type $\left(X_{5}\right)$ (1: rollable, 2 : is not rollable), transportation vehicle $\left(X_{6}\right)$ (1: palette, 2 : cloth rope, 3: chain rope), whether the part is being suitable for hooking up $\left(X_{7}\right)$ (1: yes, 2: no), welding length $\left(X_{8}\right)$. Also roughness of the part (1: rough, 2: smooth) that is measured after the grinding operations and stacking type (1: palletized, 2: unpalleted) that is appeared randomly on the online run is caused extra operations. Four grinding tables exist in the system. Since parts are processed in their separated parts (components); it is impossible to calculate the processing times on an order basis. These parts are matched according to the order numbers after the sequential grinding and painting operations are completed. Processing times are calculated for the component parts of the orders. So, the number of components and the technical specifications of these parts affect the processing times. Processing times varies according to the properties of the parts those will be processed. Random samples were taken to determine distribution of the processing times, then the probability distributions were fitted in the Arena input analyzer and chi-square goodness of fit test was conducted. The determined probability distributions that can be used for simulation are given in Table 1. According to Table 1, if a part is welded $\left(X_{2}=2\right)$, small $\left(X_{3}=1\right)$ and suitable for working on the grinding table $\left(X_{4}=1\right)$; then it can be processed on the grinding table (combination no: 1 ) with exponential (10.21) distribution, where 10.21 minutes is the mean operation time. However, the technical properties of the part such as weight, grinding type, grinding tool, transportation type, transportation vehicle, being suitable for hooking up and stacking type; do not affects the processing time of grinding on the grinding table. However, if the part is heavy $\left(X_{1}=1\right)$, welded $\left(X_{2}=2\right)$, small $\left(X_{3}=1\right)$, suitability at working on grinding table $\left(X_{4}=1\right)$ and the pallet stacking (1) is performed (combination no:3), then the processing time of transportation with transpallet is included to the simulation and calculated as triangular $(3,6,7)$ distribution, where 3,6, and 7 are the minimum, mode and maximum operation times. Processing times for other subprocesses can be interpreted similarly from the Table 1 . 
Table 1: The decision situations and their probability distributions those are used for simulation.

\begin{tabular}{|c|c|c|c|c|c|c|c|c|c|c|c|}
\hline 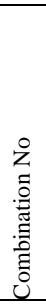 & 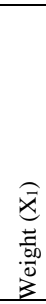 & 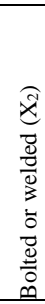 & $\begin{array}{l}\hat{x} \\
\dot{x} \\
\dot{n}\end{array}$ & 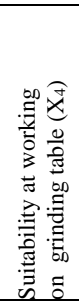 & 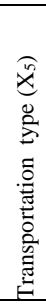 & 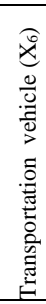 & 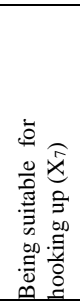 & 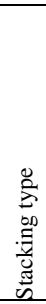 & 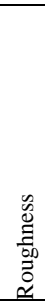 & Operation & $\begin{array}{l}\text { Probability Distribution } \\
\text { (Minutes) }\end{array}$ \\
\hline 1 & - & 2 & 1 & 1 & - & - & - & - & - & Wire grinding on the table & Exponential (10.21) \\
\hline 2 & - & 2 & 1 & 2 & - & - & - & - & - & Wire grinding on the ground & $\operatorname{Normal}(22,3.2)$ \\
\hline 3 & 1 & 2 & 1 & 1 & - & - & - & 1 & - & Transportation with transpallet & Triangular( $3,6,7)$ \\
\hline 4 & 2 & 2 & 1 & 2 & - & - & - & 1 & - & Carrying with forklift & Triangular( $(1,2,3)$ \\
\hline 5 & - & 2 & 1 & 1 & 1 & - & - & 2 & - & Rolling transport & Uniform $(2,3)$ \\
\hline 6 & - & 2 & 1 & 2 & 1 & - & - & 2 & - & Hand Carrying & Triangular $(0.5,1,2)$ \\
\hline 7 & - & 2 & 1 & - & - & - & - & - & - & Burr Control & Uniform $(4,6)$ \\
\hline 8 & - & 2 & 1 & - & - & - & - & - & 1 & Priming operation & Erlang(10.35,3) \\
\hline 9 & - & 2 & 2 & 1 & - & - & - & - & - & Wire grinding on the table & Normal(58,5.2) \\
\hline 10 & - & 2 & 2 & 2 & - & - & - & - & - & Wire grinding on the ground & $\operatorname{Normal}(180,12)$ \\
\hline 11 & - & 2 & 2 & - & 1 & - & - & - & - & Stacking parts to the palette & Triangular(4,5,7) \\
\hline 12 & - & 2 & 2 & - & 1 & - & - & - & - & Handling with forklift & Triangular( $2,4,5)$ \\
\hline 13 & - & 2 & 2 & - & 2 & - & - & - & - & Connecting ropes to pieces & Erlang $(3,2)$ \\
\hline 14 & - & 2 & 2 & - & 2 & - & - & - & - & Pulling the parts with rope & Triangular(4,7,8) \\
\hline 15 & - & 2 & 2 & - & 3 & - & - & - & - & Connecting chain to pieces & Triangular(3,5,7) \\
\hline 16 & - & 2 & 2 & - & 3 & - & - & - & - & Pulling the parts with chain & Triangular( $(8,11,12)$ \\
\hline 17 & - & 2 & 2 & - & - & - & - & - & - & Burr Control & Triangular( $3,7,8)$ \\
\hline 18 & - & 2 & 2 & - & - & - & - & - & 1 & Priming operation & Normal $(40,3.7)$ \\
\hline 19 & - & 2 & 2 & - & - & - & - & - & - & Placement of the material to the rail car & Triangular(12,14,16) \\
\hline 20 & - & 1 & - & - & - & - & - & - & 1 & Cleaning with thinner & Triangular $(5,7,8)$ \\
\hline 21 & - & 1 & - & - & - & - & - & - & 1 & Grinding & Triangular( $3,5,7)$ \\
\hline 22 & - & 1 & - & - & - & - & - & - & 1 & Final test & Triangular $(3,5,6)$ \\
\hline 23 & 1 & 1 & - & - & - & - & - & 1 & 1 & Carrying with forklift & $2.23+$ Weibul $(2.2,3.18)$ \\
\hline 24 & 2 & 1 & - & - & - & - & - & 2 & 1 & Transportation with transpallet & 4+Gamma(0.997,5.83) \\
\hline 25 & - & 1 & - & - & - & - & - & 1 & 1 & Conducting checks & 2+Lognormal(3.1,1.64) \\
\hline 26 & - & 1 & - & - & - & - & - & 1 & 1 & Placement of the material to the rail car & Triangular $(8,11,13)$ \\
\hline 27 & 1 & 1 & - & - & - & - & - & 2 & - & Hand Carrying & Triangular( $(1,3,4)$ \\
\hline 28 & 2 & 1 & - & - & - & 3 & - & 2 & - & Connecting chain to pieces & Exponential(3.05) \\
\hline 29 & 2 & 1 & - & - & - & 3 & - & 2 & - & Pulling the parts with chain & Triangular $(8,10,11)$ \\
\hline 30 & 2 & 1 & - & - & - & 2 & - & 2 & - & Connecting ropes to pieces & 9+Exponential(3.06) \\
\hline 31 & 2 & 1 & - & - & - & 2 & - & 2 & - & Pulling the parts with rope & $10+\operatorname{Erlang}(0.495,8)$ \\
\hline 32 & 2 & 1 & - & - & - & - & - & 2 & - & Burr Control & $9+8 * \operatorname{Beta}(4.09,3.7)$ \\
\hline 33 & 2 & 1 & - & - & - & - & - & 2 & 1 & Priming operation & Triangular(3,5,7) \\
\hline 34 & - & 1 & - & - & - & - & 1 & 2 & - & Hanging up the material & Triangular( $2,5,7)$ \\
\hline 35 & - & 1 & - & - & - & - & 1 & 2 & - & Placement of the material to the rail car & Erlang $(30,4)$ \\
\hline 36 & 1 & 1 & - & - & - & - & - & 1 & 2 & Carrying with forklift & Triangular(45,54,61) \\
\hline 37 & 21 & 1 & - & - & - & - & - & 1 & 2 & Transportation with transpallet & $\operatorname{Normal}(89,12)$ \\
\hline 38 & 1 & 1 & - & - & - & 3 & - & 2 & 2 & Connecting chain to pieces & $\operatorname{Normal}(87,7.2)$ \\
\hline 39 & 1 & 1 & - & - & - & 3 & - & 2 & 2 & Pulling the parts with chain & Triangular(22,28,32) \\
\hline 40 & 2 & 1 & - & - & - & 2 & - & 2 & 2 & Connecting ropes to pieces & $\operatorname{Normal}(17,4)$ \\
\hline 41 & 2 & 1 & - & - & - & 2 & - & 2 & 2 & Pulling the parts with rope & Triangular( $(11,15,17)$ \\
\hline 42 & - & 1 & - & - & - & - & - & 2 & 2 & Burr Control & Triangular $(4,8,11)$ \\
\hline 43 & - & 1 & - & - & - & - & - & 2 & 1 & Priming operation & $21+$ Exponential $(5.02)$ \\
\hline 44 & 1 & 1 & - & - & - & - & - & 2 & 2 & Placement of the material to the rail car & $\operatorname{Normal}(120,13)$ \\
\hline 45 & 1 & 1 & - & - & - & - & - & 2 & 2 & Carrying with forklift & Erlang $(20,4)$ \\
\hline 46 & 2 & 1 & - & - & - & - & 1 & - & 2 & Hanging up the material & Normal $(180,21)$ \\
\hline 47 & 2 & 1 & - & - & - & - & 2 & - & 2 & Placement of the material to the rail car & Normal $(117,15)$ \\
\hline 48 & 2 & 1 & - & - & - & - & 2 & - & 2 & Carrying with forklift & Normal $(145,12)$ \\
\hline
\end{tabular}




\subsection{Numerical Data and Results}

The data for a total of 20 orders have been gathered from the company's current waiting orders list, as listed in Table 2 and Table 3. These 20 orders correspond to the production of a total of 78 components. Four grinding tables and one painting line exist in the system. The aim here is to minimize the average of makespan of orders listed in Table 2 and Table 3 using the proposed GA-based approach under the ergonomic constraints.

Table 2: Technical specifications of orders (part 1).

\begin{tabular}{|c|c|c|c|c|c|c|c|c|c|c|c|c|c|c|c|c|c|c|c|c|c|c|c|c|c|c|c|c|c|c|c|c|c|c|c|c|}
\hline 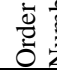 & & & $\tilde{x}$ & $\frac{m}{x}$ & $\frac{\vec{t}}{x}$ & $\frac{n}{x}$ & & $\tilde{\tilde{x}}$ & $\tilde{\tilde{x}}$ & 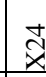 & $\ddot{\tilde{x}}$ & $\overline{\tilde{x}}$ & $\tilde{\tilde{x}}$ & $\tilde{\tilde{x}}$ & $\stackrel{t}{\tilde{x}}$ & $\tilde{\tilde{x}}$ & $\underset{\dot{x}}{\vec{x}}$ & $\frac{y}{x}$ & $\underset{⿱ 乛}{\dot{x}}$ & $\underset{x}{+}$ & $\underset{x}{x}$ & $\overline{\tilde{x}}$ & $\tilde{\varkappa}$ & $\ddot{x}$ & 苔 & $\ddot{2}$ & $\begin{array}{l}\overrightarrow{\mathbf{x}} \\
\end{array}$ & $\begin{array}{l}0 \\
\mathscr{x}\end{array}$ & $\begin{array}{l}\tilde{b} \\
\dot{x}\end{array}$ & $\underset{x}{\vec{x}}$ & $\begin{array}{l}z \\
\grave{x} \\
x\end{array}$ & $\overrightarrow{\tilde{x}}$ & $\underset{x}{\tilde{x}}$ & $\tilde{\tilde{x}}$ & $\underset{x}{x}$ & $\tilde{\tilde{x}}$ \\
\hline 1 & 3 & 1 & 1 & 2 & 0 & 0 & 1 & 3 & 2 & 0 & 0 & 1 & 2 & 1 & 0 & 0 & 1 & 1 & 2 & 0 & 0 & 1 & 2 & 1 & 0 & 0 & 1 & 3 & 2 & 0 & 0 & 1 & 2 & 1 & 0 & 0 \\
\hline 2 & 2 & 2 & 1 & 0 & 0 & 0 & 3 & 2 & 0 & 0 & 0 & 2 & 2 & 0 & 0 & 0 & 1 & 2 & 0 & 0 & 0 & 2 & 2 & 0 & 0 & 0 & 3 & 2 & 0 & 0 & 0 & 2 & 2 & 0 & 0 & 0 \\
\hline 3 & 4 & 1 & 1 & 2 & 2 & 0 & 3 & 2 & 2 & 1 & 0 & 2 & 1 & 1 & 2 & 0 & 2 & 2 & 2 & 1 & 0 & 2 & 1 & 1 & 2 & 0 & 3 & 2 & 2 & 1 & 0 & 2 & 1 & 1 & 2 & 0 \\
\hline 4 & 5 & 1 & 2 & 1 & 1 & 2 & 3 & 1 & 1 & 2 & 1 & 2 & 1 & 1 & 1 & 2 & 2 & 1 & 1 & 2 & 1 & 2 & 1 & 1 & 1 & 2 & 3 & 1 & 1 & 2 & 1 & 2 & 1 & 1 & 1 & 2 \\
\hline 5 & 3 & 1 & 1 & 1 & 0 & 0 & 3 & 3 & 2 & 1 & 2 & 1 & 2 & 2 & 0 & 0 & 2 & 2 & 2 & 1 & 2 & 1 & 2 & 2 & 0 & 0 & 3 & 3 & 2 & 1 & 2 & 1 & 2 & & 0 & 0 \\
\hline 6 & 3 & 2 & 1 & 2 & 0 & 0 & 1 & 3 & 2 & 0 & 0 & 1 & 2 & 1 & 0 & 0 & 1 & 2 & 2 & 0 & 0 & 1 & 2 & 1 & 0 & 0 & 3 & 3 & 2 & 0 & 0 & 1 & 2 & 1 & 0 & 0 \\
\hline 7 & 2 & 2 & 1 & 0 & 0 & 0 & 1 & 2 & 0 & 0 & 0 & 2 & 2 & 0 & 0 & 0 & 1 & 2 & 0 & 0 & 0 & 1 & 2 & 0 & 0 & 0 & 2 & 2 & 0 & 0 & 0 & 2 & 2 & 0 & 0 & 0 \\
\hline 8 & 4 & 2 & 1 & 1 & 1 & 0 & 2 & 1 & 2 & 2 & 0 & 2 & 1 & 1 & 2 & 0 & 1 & 2 & 2 & 1 & 0 & 1 & 1 & 1 & 2 & 0 & 2 & 2 & 2 & 1 & 0 & 1 & 1 & 1 & 2 & 0 \\
\hline 9 & 5 & 1 & 2 & 1 & 2 & 2 & 3 & 1 & 1 & 2 & 3 & 2 & 1 & 1 & 1 & 1 & 1 & 1 & 1 & 2 & 2 & 2 & 1 & 1 & 2 & 1 & 1 & 1 & 1 & 2 & 1 & 1 & 2 & 2 & 1 & 2 \\
\hline 10 & 3 & 1 & 1 & 1 & 0 & 0 & 3 & 3 & 2 & 3 & 2 & 2 & 1 & 1 & 0 & 0 & 2 & 1 & 2 & 1 & 1 & 1 & 2 & 1 & 0 & 0 & 3 & 3 & 2 & 1 & 2 & 2 & 2 & 1 & 0 & 0 \\
\hline 11 & 3 & 2 & 2 & 2 & 0 & 0 & 1 & 3 & 2 & 0 & 0 & 1 & 1 & 1 & 0 & 0 & 2 & 1 & 2 & 0 & 0 & 2 & 2 & 2 & 0 & 0 & 1 & 3 & 2 & 0 & 0 & 2 & 2 & 1 & $\underline{0}$ & 0 \\
\hline 12 & 2 & 1 & 1 & 0 & 0 & 0 & 2 & 3 & 0 & 0 & 0 & 2 & 2 & 0 & 0 & 0 & 2 & 2 & 0 & 0 & 0 & 1 & 2 & 0 & 0 & 0 & 3 & 2 & 0 & 0 & 0 & 2 & 2 & 0 & 0 & 0 \\
\hline 13 & 4 & 1 & 1 & 2 & 2 & 0 & 1 & 2 & 2 & 2 & 0 & 1 & 2 & 2 & 2 & 0 & 1 & 2 & 2 & 1 & 0 & 1 & 1 & 2 & 1 & 0 & 3 & 2 & 1 & 2 & 0 & 1 & 2 & 2 & 1 & 0 \\
\hline 14 & 5 & 1 & 2 & 1 & 2 & 1 & 3 & 1 & 1 & 2 & 2 & 1 & 1 & 1 & 1 & 2 & 1 & 1 & 1 & 2 & 2 & 2 & 1 & 1 & 1 & 1 & 2 & 1 & 2 & 3 & 2 & 1 & 1 & 2 & 2 & 2 \\
\hline 15 & 3 & 2 & 1 & 1 & 0 & 0 & 3 & 3 & 2 & 1 & 3 & 1 & 1 & 2 & 0 & 0 & 1 & 2 & 2 & 1 & 2 & 1 & 1 & 1 & 0 & 0 & 3 & 3 & 3 & 2 & 3 & 1 & 1 & 1 & 0 & 0 \\
\hline 16 & 3 & 2 & 2 & 2 & 0 & 0 & 1 & 3 & 2 & 0 & 0 & 1 & 1 & 2 & 0 & 0 & 1 & 2 & 2 & 0 & 0 & 2 & 1 & 2 & 0 & 0 & 1 & 2 & 3 & 0 & 0 & 1 & 2 & 1 & 0 & 0 \\
\hline 17 & 2 & 1 & 1 & 0 & 0 & 0 & 2 & 3 & 0 & 0 & 0 & 2 & 2 & 0 & 0 & 0 & 1 & 1 & 0 & 0 & 0 & 2 & 2 & 0 & 0 & 0 & 3 & 3 & 0 & 0 & 0 & 1 & 1 & 0 & 0 & 0 \\
\hline 18 & 4 & 1 & 1 & 2 & 2 & 0 & 3 & 2 & 2 & 1 & 0 & 2 & 2 & 2 & 2 & 0 & 2 & 2 & 2 & 1 & 0 & 1 & 1 & 1 & 1 & 0 & 3 & 2 & 2 & 1 & 0 & 2 & 1 & 1 & 1 & 0 \\
\hline 19 & 5 & 2 & 2 & 1 & 1 & 1 & 3 & 1 & 1 & 2 & 2 & 1 & 1 & 1 & 1 & 2 & 2 & 1 & 1 & 2 & 2 & 2 & 1 & 1 & 2 & 2 & 3 & 1 & 1 & 2 & 3 & 2 & 1 & 1 & 1 & 1 \\
\hline 20 & 3 & 2 & 2 & 1 & 0 & 0 & 3 & 3 & 2 & 1 & 2 & 1 & 2 & 2 & 0 & 0 & 1 & 2 & 2 & 1 & 1 & 1 & 2 & 2 & 0 & 0 & 3 & 3 & 2 & 1 & 2 & 1 & 2 & 2 & 0 & 0 \\
\hline
\end{tabular}

Table 3. Technical specifications of orders (part 2).

\begin{tabular}{|l|l|l|l|l|l|l|l|l|l|l|l|l|l|l|l|l|l|l|l|l|}
\hline Order Number & 1 & 2 & 3 & 4 & 5 & 6 & 7 & 8 & 9 & 10 & 11 & 12 & 13 & 14 & 15 & 16 & 17 & 18 & 19 & 20 \\
\hline $\begin{array}{l}\text { Number of } \\
\text { Components }\end{array}$ & 3 & 2 & 4 & 5 & 3 & 3 & 2 & 4 & 5 & 3 & 3 & 2 & 4 & 5 & 3 & 3 & 2 & 4 & 5 & 3 \\
\hline $\mathrm{X} 81$ & 100 & 400 & 600 & 1000 & 1600 & 200 & 500 & 567 & 4555 & 4324 & 123 & 234 & 567 & 654 & 345 & 789 & 876 & 543 & 457 & 654 \\
\hline $\mathrm{X} 82$ & 110 & 410 & 610 & 1010 & 1610 & 110 & 410 & 610 & 1010 & 1610 & 110 & 410 & 610 & 1010 & 1610 & 110 & 410 & 610 & 1010 & 1610 \\
\hline $\mathrm{X} 83$ & 120 & 0 & 620 & 1020 & 1620 & 120 & 0 & 620 & 1020 & 1620 & 120 & 0 & 620 & 1020 & 1620 & 120 & 0 & 620 & 1020 & 1620 \\
\hline $\mathrm{X} 84$ & 0 & 0 & 0 & 1030 & 0 & 0 & 0 & 0 & 1030 & 0 & 0 & 0 & 0 & 1030 & 0 & 0 & 0 & 0 & 1030 & 0 \\
\hline $\mathrm{X} 85$ & 0 & 0 & 0 & 1040 & 0 & 0 & 0 & 0 & 1040 & 0 & 0 & 0 & 0 & 1040 & 0 & 0 & 0 & 0 & 1040 & 0 \\
\hline
\end{tabular}


Constraints related with the technical design parameters of the orders, number of manufacturing resources, capacities of the resources and etc. affect the average makespan of the waiting orders under the dynamic manufacturing environment and studied in the previous studies in the literature. However, ergonomic constraints are another important constraints that should not be ignored in labor intensive project type work. There is an unplanned break in the labor-intensive work that is done with an inappropriate work posture, which is called a masked rest and is done by the employee to rest his muscles between the two works. Under the dynamic workshop conditions, cumulative sum of these small resting times affects the makespan. The grinding process is carried out by four female workers whose weights range from 60 to $70 \mathrm{~kg}$. One shift per day is 8 hours. Grinding of heavy parts is done on the ground by leaning and this process is evaluated within the scope of heavy work. According to the nature of the operation (work on ground, heavy, etc.), ergonomic constraint-related processing times are expected to increase. The changes considered in the calculations according to the ergonomic constraints are as follows. The grinding to be done on large parts is done on the ground because of the large and heavy part. The employee works by leaning and squatting in the grinding process. This working posture has been analyzed with Anybody Computer Assisted Ergonomics software and it has been found that it causes difficulty in the employee's waist, legs, arms and neck. For this reason, it has been observed that employees are given masked rests during the subsequent grinding operations on the ground. According to the previous observations, the time interval for the masked resting between successive grinding operations of two parts fits to the triangular distribution $(1,1.5,2)$ in minutes. If a third part is added then the probability distribution of masked rests is calculated as triangular distribution $(1.5,2,2.5)$ in minutes. More than 3 consecutive grinding operations that had to be done on the ground is not observed in this case study, but if such a situation occurs in simulations, it is assumed that the latency period between transactions is again triangular $(1.5,2.2,5)$. One of the objectives of the work is to determine the optimized order sequence those are waiting to be processed, so as to minimize the total anticipated masked rest time periods.

Firstly, the given initial order (1-2-3-4-5-6-7-8-9-10-11-12-13-14-15-16-17-18-19-20) is simulated by 100 numbers of replication with both ergonomic constraints and without ergonomic constraints. It is calculated by simulation the average makespan of given order with ergonomic constraints is 24092.58 minutes and the one without ergonomic constraints is 23850.13 minutes. As a result, ergonomic constraints have a significant effect on the average makespan. Then, the GA is run with parameters PopSize $=25$, MaxNbIt $=40, \mathrm{cr}=0.5$, and $m r=0.1$ and a total of 625 chromosomes have been generated during the algorithm run. The chromosomes that completion time exceeds the delivery time are eliminated from the population. The individual which gives the best FV (23972.43 minutes) is 19-13-6-7-15-8-17-5-10-20-11-9-4-2-12-16-183-1-14. As seen from these results, the average makespan under ergonomic constraints is reduced from 24092.58 to 23972.43 minutes. The $\% 95$ confidence interval for order sequence generated by GA is calculated [23905.81;24038.19]. However, the $\% 95$ confidence interval for given initial order sequence is calculated [24043.88;24141.28]. These confidence intervals show that order sequence generated by GA outperforms statistically the given initial order sequence at the $\% 95$ confidence level.

\section{Conclusions}

This study focused on determining the best possible processing sequence of the orders to minimize the average makespan by discussing the dynamic job-shop conditions, technical specifications of orders and ergonomic constraints related with these specifications. This study makes significant contributions to knowledge. Existing researches in the literature have not directly considered the technical design specifications of the orders and ergonomic constraints together in order scheduling. However, in this study, in addition to the job-shop characteristics of the production environment, the technical specifications of the orders and the related ergonomic constraints are directly considered in scheduling orders. Also, GA and simulation are integrated to obtain a feasible as well as powerful solution in sequencing customer orders. The result of the case study made it clear that the average makespan is reduced by proposed methodology. For future research additional ergonomic constraints will be added to the simulation model for better estimation.

\section{Acknowledgements}

The authors would gratefully like to thank YEMTAR Research \& Development Department for its valuable supports lead to reveal this paper. 


\section{References}

[1] M. A. B. Ocaktan, I. Kucukkoc, A. D. Karaoglan, A. Cicibas and K. Buyukozkan, "Scheduling Customized Orders: A Case Study at BEST Transformers Company," $6^{\text {th }}$ International Conference on Mechanics and Industrial Engineering (ICMIE'17), Paper ID: ICMIE 118, pp. 1-8, Rome, Italy, 2017.

[2] A. D. Karaoglan and O. Karademir, "Flow time and product cost estimation by using an artificial neural network (ANN): A case study for transformer orders," Engineering Economist, 2016. DOI:10.1080/0013791X. 2016.1185808).

[3] A. D. Karaoglan, M. A. B. Ocaktan and A. Cicibas, "Flow Time and Due Date Estimation for Customer Orders According to Design Criteria: A Case Study of BEST Transformers Company," 4th Annual International Conference on Industrial, Systems and Design Engineering, Athens, Greece, vol. IND2016-1974, 2016.

[4] A. D. Karaoglan, "Residual based flow time estimation algorithm for labor intensive project type production systems," Dokuz Eylul University Faculty of Engineering, Journal of Science and Eng., vol. 18, no. 3, pp. 580-595, 2016.

[5] D. Krige, J. Bekker and C. Schutte, "Simulation-based online scheduling in a make-to-order job shop," South African Journal of Industrial Engineering, vol. 20, no. 2, pp. 205-217, 2009.

[6] R. Tavakkoli-Moghaddam and M. Daneshmand-Mehr, "A computer simulation model for job shop scheduling problems minimizing makespan," Computers \& Industrial Engineering, vol. 48, no. 4, pp. 811-823, 2005.

[7] B. S. de Ugarte, R. Pellerin and A. Artiba, "An improved genetic algorithm approach for on-line optimization problems," Production Planning\&Control, vol. 22, no. 8, pp. 742-753, 2011.

[8] Z. Jia, X. Lu, J. Yang and D. Jia, "Research on job-shop scheduling problem based on genetic algorithm," International Journal of Production Research, vol. 49, no. 12, pp. 3585-3604, 2011.

[9] R. Qing-dao-er-ji and Y. Wang, "A new hybrid genetic algorithm for job shop scheduling problem," Computers\&Operations Research, vol. 39, no. 10, pp. 2291-2299, 2012.

[10] X. W. Huang, X. Y. Zhao and X. L. Ma, "An improved genetic algorithm for job-shop scheduling problem with process sequence flexibility," International Journal of Simulation Modelling, vol. 13, no. 4, pp. 510-522, 2014.

[11] H. Zupan, N. Herakovic, M. Starbek and J. Kusar, "Hybrid algorithm based on priority rules for simulation of workshop production," International Journal of Simulation Modelling, vol. 15, no. 1, pp. 29-41, 2016.

[12] A. Baykasoglu, S. Ozmehmet Tasan, A. S. Tasan and S. Demirkol Akyol, "Modeling and solving assembly line design problems by considering human factors with a real-life application," Human Factors and Ergonomics in Manufacturing \& Service Industries, vol. 27, no. 2, pp. 96-115, 2017.

[13] S. Demirkol Akyol and A. Baykasoğlu, "ErgoALWABP: a multiple-rule based constructive randomized search algorithm for solving assembly line worker assignment and balancing problem under ergonomic risk factors," Journal of Intelligent Manufacturing, 2016. DOI 10.1007/s10845-016-1246-6.

[14] B. J. Carnahan, M. S. Redfern and B. Norman, "Designing safe job rotation schedules using optimization and heuristic search," Ergonomics, no. 43, pp. 543-560, 2000.

[15] T. Kullpattaranirun and S. Nanthavanij, "A heuristic genetic algorithm for solving complex safety-based work assignment problems," The International Journal of Industrial Engineering: Theory, Applications and Practice, vol. 12, pp. 45-57, 2005.

[16] M. B. Aryanezhad, V. Kheirkhah, V. Deljoo, Mirzapour and SMJ. Al-e-hashem, "Designing safe job rotation schedules based upon workers skills," International Journal of Advanced Manufacturing Technology, vol. 41, pp. 193-199, 2009.

[17] Y. Kara, Y. Atasagun, H. Gokcen, S. Hezer and N. Demirel, "An integrated model to incorporate ergonomics and resource restrictions into assembly line balancing," International Journal of Computer Integrated Manufacturing, vol. 27, pp. 997-1007, 2014.

[18] J. E. Biegel and J. J. Davern, "Genetic algorithms and job shop scheduling," Computers \& Industrial Engineering, vol. 19, no. 1-4, pp. 81-91, 1990.

[19] J. F. Goncalves, J. J. de Magalhães Mendes and M. G. C. Resende, "A hybrid genetic algorithm for the job shop scheduling problem", European Journal of Operational Research, vol. 167, no. 1, pp. 77-95, 2005.

[20] J. H. Holland, Adaptation in Natural and Artificial Systems. Cambridge, MIT Press, 1975.

[21] D. E. Goldberg, Genetic Algorithms in Search,Optimization and Machine Learning. Boston, MA, USA: AddisonWesley Longman Publishing Co. Inc. 1989.

[22] M. D. Rosetti, Simulation Modeling and Arena, 2nd ed. New Jersey: John Wiley\&Sons Inc. 2010. 